\title{
An Experiment with Fuzzy Sets in Data Mining
}

\author{
David L. Olson ${ }^{1}$, Helen Moshkovich ${ }^{2}$, and Alexander Mechitov \\ ${ }^{1}$ University of Nebraska, Department of Management, Lincoln, NE USA 68588-0491 \\ dolson3@unl. edu \\ ${ }^{2}$ Montevallo University, Comer Hall, Montevallo, AL USA 35115 \\ MoshHMamontevallo.edu, Mechitov@montevallo.edu
}

\begin{abstract}
Fuzzy modeling provides a very useful tool to deal with human vagueness in describing scales of value. This study examines the relative error in decision tree models applied to a real set of credit card data used in the literature, comparing crisp models with fuzzy decision trees as applied by See5, and as obtained by categorization of data. The impact of ordinal data is also tested. Modifying continuous data was expected to degrade model accuracy, but was expected to be more robust with respect to human understanding. The degree of accuracy lost by See5 fuzzification was minimal (in fact more accurate in terms of total error), although bad error was worse. Categorization of data yielded greater inaccuracy. However, both treatments are still useful if they better reflect human understanding. An additional conclusion is that when categorizing data, care should be taken in setting categorical limits.
\end{abstract}

Keywords: Decision tree rules, fuzzy data, ordinal data.

\section{Introduction}

Classification tasks in business applications may be viewed as tasks with classes reflecting the levels of the same property. Evaluating creditworthiness of clients is rather often measured on an ordinal level as, e.g., $\{$ excellent $\},\{$ good $\},\{$ acceptable $\}$, or \{poor $\}$ (Ben David et al., 1989.) Applicants for a job are divided into accepted and rejected, but sometimes there may be also a pool of applicants left for further analysis as they may be accepted in some circumstances [2], [11]. Different cars may be divided into groups $\{$ very good $\}$, \{good $\},\{$ acceptable $\},\{$ unacceptable $\}$. This type of tasks is called "ordinal classification" [5]. The peculiarity of the ordinal classification is that data items with \{better\} qualities (characteristics) logically are to be presented in \{better\} classes: the better the article in its characteristics the closer it is to the class \{accepted . It was shown in[6] that taking into account possible ordinal dependence between attribute values and final classes may lead to a smaller number of rules with the same accuracy and enable the system to extend obtained rules to instances not presented in the training data set.

There are many data mining tools available, to cluster data, to help analysts find patterns, to find association rules. The majority of data mining approaches to classification tasks, work with numerical and categorical information. Not many data mining techniques take into account ordinal data features. 
Real-world application is full of vagueness and uncertainty. Several theories on managing uncertainty and imprecision have been advanced, to include fuzzy set theory [13], probability theory [8], rough set theory [7] and set pair theory [14], [15]. Fuzzy set theory is used more than the others because of its simplicity and similarity to human reasoning. Although there is a wide variety of different approaches within this field, many view advantages of fuzzy approach in data mining as an ..."interface between a numerical scale and a symbolic scale which is usually composed of linguistic terms" [4].

Fuzzy association rules described in linguistic terms help increase the flexibility for supporting users in making decisions. Fuzzy set theory is being used more and more frequently in intelligent systems. A fuzzy set $A$ in universe $U$ is defined as $A=\left\{\left(x, \mu_{A}(x)\right) \mid x \in U, \mu_{A}(x) \in[0,1]\right\}$, where $\mu_{A}(x)$ is a membership function indicating the degree of membership of $x$ to $A$. The greater the value of $\mu_{A}(x)$, the more $x$ belongs to $A$. Fuzzy sets can also be thought of as an extension of the traditional crisp sets and categorical/ordinal scales, in which each element is either in the set or not in the set (a membership function of either 1 or 0 .)

Fuzzy set theory in its many manifestations (interval-valued fuzzy sets, vague sets, grey-related analysis, rough set theory, etc.) is highly appropriate for dealing with the masses of data available. This paper will review some of the general developments of fuzzy sets in data mining, with the intent of seeing some of the applications in which they have played a role in advancing the use of data mining in many fields. It will then review the use of fuzzy sets in two data mining software products, and demonstrate the use of data mining in an ordinal classification task. The results will be analyzed through comparison with the ordinal classification model. Possible adjustments of the model to take into account fuzzy thresholds in ordinal scales will be discussed.

\section{Fuzzy Set Experiments in See5}

See5, a decision tree software, allows users to select options to soften thresholds through selecting a fuzzy option. This option would insert a buffer at boundaries (which is how PolyAnalyst works as well). The buffer is determined by the software based on analysis of sensitivity of classification to small changes in the threshold. The treatment from there is crisp, as opposed to fuzzy. Thus, in decision trees, fuzzy implementations seem to be crisp models with adjusted set boundaries.

See5 software was used on a real set of credit card data[10]. This dataset had 6,000 observations over 64 variables plus an outcome variable indicating bankruptcy or not (variables defined in [10]). Of the 64 independent variables, 9 were binary and 3 categorical. The problem can be considered to be an ordinal classification task as the two final classes are named as "GOOD" and "BAD" with respect to financial success. This means that majority of the numerical and categorical attributes (including binary ones) may be easily characterized by more preferable values with respect to "GOOD" financial success.

The dataset was balanced to a degree, so that it contained 960 bankrupt outcomes ("BAD”) and 5040 not bankrupt (“GOOD.") Winnowing was used in See5, which 
reduced the number of variables used in models to about 20 . Using 50 percent of the data for training, See5 selected 3000 observations at random as the training set, which was then tested on the remaining 3000 observations in the test set. Minimum support on See 5 was varied over the settings of 10, 20, and 30 cases. Pruning confidence factors were also varied, from $10 \%$ (greater pruning), $20 \%, 30 \%$, and $40 \%$ (less pruning). Data was locked within nominal data runs, so that each treatment of pruning and minimum case settings was applied to the same data within each repetition. Five repetitions were conducted (thus there were 12 combinations repeated five times, or 60 runs). Each run was replicated for original crisp data, original data using fuzzy settings, ordinal crisp data, ordinal data using fuzzy settings, and categorical data (See5 would have no difference between crisp and fuzzy settings). Rules obtained were identical across crisp and fuzzy models, except fuzzy models had adjusted rule limits. For instance, in the first run, the following rules were obtained:

\begin{tabular}{|c|c|c|c|}
\hline RISP MODE & $\begin{array}{l}\text { RULE 1: } \\
\text { RULE 2: } \\
\text { RULE 3: }\end{array}$ & $\begin{array}{l}\text { IF RevtoPayNov } \leq 11.441, \\
\text { IF RevtoPayNov }>11.441 \text { AND } \\
\text { IF CoverBal3 }=1 \\
\text { IF RevtoPayNov }>11.441 \text { AND } \\
\text { IF CoverBal3 =0 AND } \\
\text { IF OpentoBuyDec }>5.35129 \\
\text { IF RevtoPayNov }>11.441 \text { AND } \\
\text { IF CoverBal3 =0 AND } \\
\text { IF OpentoBuyDec } \leq 5.35129 \text { AND } \\
\text { IF NumPurchDec } \leq 2.30259\end{array}$ & $\begin{array}{l}\text { then GOOD } \\
\text { then BAD } \\
\text { GOOD }\end{array}$ \\
\hline
\end{tabular}

The fuzzy model for this data set:

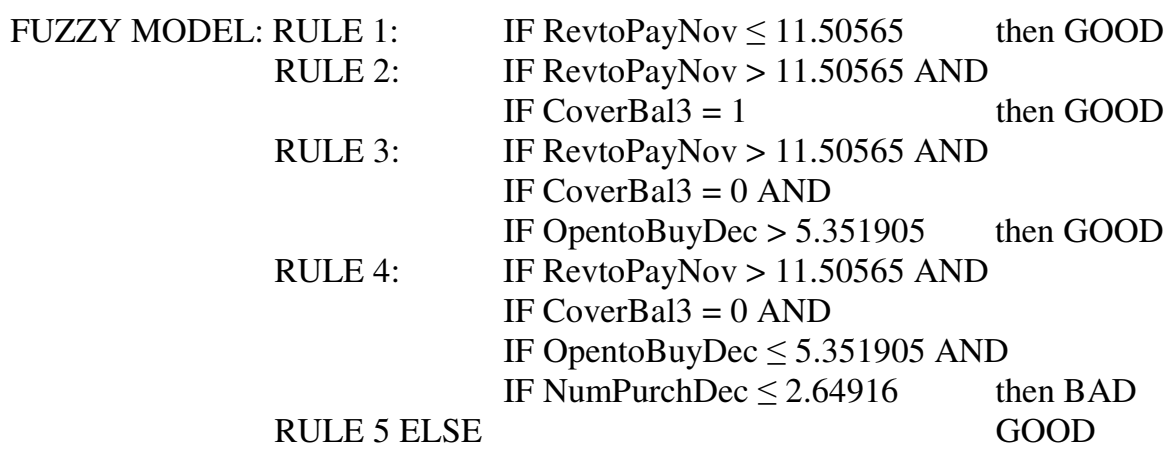

Binary and categorical data are not affected by the fuzzy option in See5. They are considered already "fuzzified" with several possible values and corresponding membership function of 1 and 0 .

Models run with initial data (numeric and categorical scales) in original crisp form, and using See5's fuzzification were obtained. There were 15 runs averaged for each pruning level, and 20 runs averaged for each minimum case level. The overall line is 
based on all 60 runs. The average number of crisp rules was 9.4 (fuzzy rules were the same, with different limits). Crisp total error averaged 488.7, while fuzzy total error averaged 487.2.

The number of rules responded to changes in pruning rates and minimum case settings as expected (the tie between 20 percent and 30 percent pruning rates can be attributed to data sampling chance). There were no clear patterns in error rates by treatment. Fuzzy models were noticeably different from crisp models in that they had higher error rates for bad cases, with corresponding improvement in error in the good cases. The overall error was tested by t-test, and the only significant differences found were that the fuzzy models had significantly greater bad error than the crisp models, and significantly less cheap error. The fuzzy models had slightly less overall average error, but given the context of credit cards, bad error is much more important. For data fit, here the models were not significantly different. For application context, the crisp models would clearly be preferred. The generalizable conclusion is not that crisp models are better, only that in this case the fuzzy models were worse, and in general one cannot count on the same results across crisp and fuzzified models.

In this case introducing fuzzy thresholds in the rules did not lead to any significant results. The usage of small fuzzy intervals instead of crispy thresholds did not significantly improve the accuracy of the model and did not provide better interpretation of the introduced "interval rules." On the other hand, crisp data was not significantly better than the fuzzy data.

The same tests were conducted with presenting relevant binary and categorical variables in an ordinal form. See5 allows stating that the categorical scale is "[ordered]" with the presented order of attribute values corresponding to the order of final classes. The order is not derived from the data but is introduced by the user as a pre-processing step in rules/tree formation.

See5 would not allow locking across data sets, and required different setup for ordinal specification, so we could not control for data set sampling across the tests. Some categorical and/or binary variables such as "Months late" were clearly ordinal and were marked as ordinal for this experiment. Categorical variables with no clear ordinal qualities such as "State," were left nominal. Crisp rules averaged 7.0, with total error of 487. Fuzzy total error was 482.

The number of rules clearly dropped. Expected response of number of rules to pruning and minimum case settings behaved as expected, with the one anomaly at 20 percent pruning, again explainable by the small sample size. Total error rates within ordinal model were similar to the nominal case in the first set of runs, with fuzzy model total error rates showing up as slightly significant ( 0.086 error probability) in the ordinal models.

Comparing nominal and ordinal models, the number of rules was significantly lower for ordinal models (0.010 error probability.) There were no significances in errors across the two sets except for total error (ordinal models had slightly significantly lower total errors, with 0.087 error probability.) This supports our previous finding that using ordinal scales where appropriate lead to a set of more interesting rules without loss in accuracy [6].

The data was categorized into 3 categories for each continuous variable. This in itself is another form of fuzzification. Twenty five variables were selected based upon the typical winnowing results of the original data. The same tests were conducted, 
although fuzzification was not used since See5 would have no difference in applying its fuzzification routine (we did run as a check, but results were always identical). Average number of rules was just over 6, with average total error 495.7. Results were much worse than the results obtained in prior runs with continuous and ordinal data. That is clearly because in the third set of runs, data was categorized manually, while in the prior two runs See5 software set the categorical limits. The second set of runs involved data converted to fuzzy form by the See5 software. These are two different ways to obtain fuzzy categories. Clearly the software can select cutoff limits that will outperform ad hoc manual cutoffs.

\section{Fuzzy Sets and Ordinal Classification Task}

Previous experiments showed very modest improvements in the rule set derived from introducing of fuzzy intervals instead of crisp thresholds for continuous scales using SEE5. Interpretation of the modified rules was not "more friendly" or "more logical." Using stable data intervals was in general slightly more robust than using crisp thresholds. Considering ordinal properties of some categorical/binary attributes led to a better rule set although this did not change the fuzzy intervals for the continuous scales. This supports our previous findings [6].

One of the more useful aspects of fuzzy logic may be the orientation on the partition of continuous scales into a pre-set number of "linguistic summaries"[12]. In [1] this approach is used to form fuzzy rules in a classification task. The main idea of the method is to use a set of pre-defined linguistic terms for attributes with continuous scales (e.g., "Young", "Middle", "Old" for an attribute "Age" measured continuously). In this approach. the traditional triangular fuzzy number is calculated for each instance of age in the training data set, e.g. age 23 is presented in "Young" with a 0.85 membership function and in "Middle" with a 0.15 membership function ( 0 in "Old"). Thus the rewritten data set is used to mine interesting IF-THEN rules using linguistic terms.

One of the advantages of the proposed approach stressed by the authors is the ability of the mining method to produce rules useful for the user. In [3], the method was used to mine a database for direct marketing campaign of a charitable organization. In this case the domain expert defined appropriate uniform linguistic terms for quantitative attributes. For example, an attribute reflecting the average amount of donation (AVGEVER) was fuzzified into "Very low" (0 to \$300), "Low" (\$100 to \$500), "Medium" (\$300 to \$700), "High" (\$500 to \$900) and "Very High" (over \$700). The analogous scale for frequency of donations (FREQEVER) was presented as follows: "Very low" (0 to 3), "Low" (1 to 5), "Medium" (3 to 7), "High" (5 to 9) and "Very High" (over 7). Triangular fuzzy numbers were derived from these settings for rule mining. The attribute to be predicted was called "Response to the direct mailing" and included two possible values "Yes" and "No." The database included 93 attributes, 44 having continuous scales.

Although the application of the method produced a huge number of rules $(31,865)$ with relatively low classification accuracy (about 64\%), the authors argued that for a task of such complexity the selection of several useful rules by the user of the results was enough to prove the usefulness of the process. The presented rules found useful by the user were presented as follows: 
Rule 1: IF a donor was enrolled in any donor activity in the past (ENROLL $=Y E S$ ), THEN he/she will have RESPONSE $=Y E S$

Rule 2: IF a donor was enrolled in any donor activity in the past (ENROLL $=Y E S$ ) AND did not attend it $(\mathrm{ATTENDED}=N O)$, THEN he/she will have $\mathrm{RESPONSE}=Y E S$

Rule 3: IF FREQEVER = MEDIUM, THEN RESPONSE $=Y E S$

Rule 4: IF FREQEVER $=H I G H$, THEN RESPONSE $=Y E S$

Rule 5: IF FREQEVER = VERY HIGH, THEN RESPONSE=YES

We infer two conclusions based on these results. First, if obvious ordinal dependences between final classes of RESPONSE (YES/NO) and such attributes as ENROLL, ATTENDED, and FREQEVER were taken into account the five rules could be collapsed into two without any loss of accuracy and with higher levels for measures of support and confidence: rule 1 and a modified rule 3 in the following format "IF FREQEVER is at least MEDIUM, THEN RESPONSE=YES." Second, although presented rules are "user friendly" and easily understandable, they are not as easily applicable. Overlapping scales for FREQEVER makes it difficult for the user to apply the rules directly. It is necessary to carry out one more step - agree on the number where "medium" frequency starts (if we use initial database) or a level of a membership function to use in selecting "medium" frequency if we use the "rewritten" dataset. The assigned interval of 3 to 5 evidently includes "High" frequency (which does not bother us) but also includes "Low" frequency" which we possibly would not like to include into our mailing list. As a result a convenient approach for expressing continuous scales with overlapping intervals at the preprocessing stage may be not so convenient in applying simple rules.

This presentation of the ordinal classification task allows use of this knowledge to make some additional conclusions about the quality of the training set of objects. Ordinal classification allows introduction of the notion of the consistency of the training set as well as completeness of the training set. In the case of the ordinal classification task quality of consistency in a classification (the same quality objects should belong to the same class) can be essentially extended: all objects with higher quality among attributes should belong to a class at least as good as objects with lower quality. This condition can be easily expressed as follows: if $A_{i}(x) \geq A_{i}(y)$ for each $\mathrm{i}=1,2, \ldots, \mathrm{p}$, then $\mathrm{C}(\mathrm{x}) \geq \mathrm{C}(\mathrm{y})$.

We can also try to evaluate representativeness of the training set by forming all possible objects in $U$ (we can do that as we have a finite number of attributes with a small finite number of values in their scales) and check on the proportion of them presented in the training set. It is evident that the smaller this proportion the less discriminating power we'll have for the new cases. We can also express the resulting rules in a more summarized form by lower and upper border instances for each class [6].

Advantages of using ordinal scales in an ordinal classification task do not lessen advantages of appropriate fuzzy set techniques. Fuzzy approaches allow softening strict limitations of ordinal scales in some cases and provides a richer environment for data mining techniques. On the other hand, ordinal dependences represent essential domain knowledge which should be incorporated as much as possible into the mining process. In some cases the overlapping areas of attribute scales may be resolved by introducing additional linguistic ordinal levels. For example, we can introduce an 
ordinal scale for age with the following levels: "Young" (less than 30), "Between young and medium" (30 to 40), "Medium" (40 to 50), "Between medium and old" (50 to 60) and "Old" (over 60). Though it will increase the dimensionality of the problem, it would provide crisp intervals for the resulting rules.

Ordinal scales and ordinal dependences are easily understood by humans and are attractive in rules and explanations. These qualities should be especially beneficial in fuzzy approaches to classification problems with ordered classes and "linguistic summaries" in the discretization process. The importance of ordinal scales for data mining is evidenced by appearance of this option in many established mining techniques. See5 includes the variant of ordinal scales in the problem description [9].

\section{Conclusions}

Fuzzy representation is a very suitable means for humans to express themselves. Many important business applications of data mining are appropriately dealt with by fuzzy representation of uncertainty. We have reviewed a number of ways in which fuzzy sets and related theories have been implemented in data mining. The ways in which these theories are applied to various data mining problems will continue to grow.

Ordinal data is stronger than nominal data. There is extra knowledge in knowing if a greater value is preferable to a lesser value (or vice versa). This extra information can be implemented in decision tree models, and our results provide preliminary support to the idea that they might strengthen the predictive power of data mining models.

Our contention is that fuzzy representations better represent what humans mean. Our brief experiment was focused on how much accuracy was lost by using fuzzy representation in one application - classification rules applied to credit applications. While we expected less accuracy, we found that the fuzzy models (as applied by See5 adjusting rule limits) usually actually were more accurate. Models applied to categorical data as a means of fuzzification turned out less accurate in our small sample. While this obviously cannot be generalized, we think that there is a logical explanation. While fuzzification will not be expected to yield better fit to training data, the models obtained by using fuzzification will likely be more robust, which is reflected in potentially equal if not better fit on test data. The results of these preliminary experiments indicate that implementing various forms of fuzzy analysis will not necessarily lead to reduction in classification accuracy.

\section{References}

1. Au, W-H, Keith C. C. Chan: Classification with Degree of Membership: A Fuzzy Approach. ICDM (2001): 35-42

2. David, B. A. (1992): Automated generation of symbolic multiattribute ordinal knowledgebased DSSs: Methodology and applications. Decision Sciences, 23(6), 157-1372

3. Chan, Keith C. C., Wai-Ho Au, Berry Choi: Mining Fuzzy Rules in A Donor Database for Direct Marketing by a Charitable Organization. IEEE ICCI (2002): 239-246 
4. Dubois, D., E. Hullermeier, H. Prade: A Systematic Approach to the Assessment of Fuzzy Association Rules. Data Mining and Knowledge Discovery, (2006), July, 1-26

5. Larichev, O.I., Moshkovich, H.M. (1994): An approach to ordinal classification problems. International Trans. on Operations Research, 82, 503-521

6. Moshkovich H.M., Mechitov A.I., Olson, D.: Rule Induction in Data Mining: Effect of Ordinal Scales. Expert Systems with Applications Journal, (2002), 22, 303-311

7. Pawlak, Z.: Rough set, International Journal of Computer and Information Sciences. (1982), 341-356

8. Pearl, J.: Probabilistic reasoning in intelligent systems, Networks of Plausible inference, Morgan Kaufmann, San Mateo,CA (1988)

9. See5 - http://www.rulequest.com

10. Shi, Y., Peng, Y., Kou, G., Chen, Z.: Classifying credit card accounts for business intelligence and decision making: A multiple-criteria quadratic programming approach, International Journal of Information Technology \& Decision Making 4:4 December (2005), 581-599

11. Slowinski, R. (1995): Rough set approach to decision analysis. AI Expert,19-25

12. Yager, R.R.: On Linguistic Summaries of Data, in G. Piatetsky-Shapiro and W.J. Frawley 9Eds.) Knowledge Discovery in Databases, Mento Park, CA: AAAI/MIT Press, (1991), 347-363

13. Zadeh, L.A.: Fuzzy sets, Information and Control 8 (1965), 338-356

14. Zhao, K.-G.: Set pair analysis - a new concept and new systematic approach. Proceedings of national system theory and regional analysis conference, Baotou (1989) (In Chinese)

15. Zhao, K.-G.: Set pair analysis and its preliminary application, Zhejiang Science and Technology Press (2000) (In Chinese) 